\title{
Evaluation of a highly refined prediction model in knowledge-based volumetric modulated arc therapy planning for cervical
} cancer

Mingli Wang ${ }^{1,2,3 \dagger}$, Huikuan $\mathrm{Gu}^{1,2,3+}$, Jiang Hu${ }^{1,2,3+}$, Jian Liang ${ }^{1,2,3}$, Sisi $\mathrm{Xu}^{1,2,3}$ and Zhenyu Qi ${ }^{1,2,3^{*}}$

\begin{abstract}
Background and purpose: To explore whether a highly refined dose volume histograms (DVH) prediction model can improve the accuracy and reliability of knowledge-based volumetric modulated arc therapy (VMAT) planning for cervical cancer.
\end{abstract}

Methods and materials: The proposed model underwent repeated refining through progressive training until the training samples increased from initial 25 prior plans up to 100 cases. The estimated DVHs derived from the prediction models of different runs of training were compared in 35 new cervical cancer patients to analyze the effect of such an interactive plan and model evolution method. The reliability and efficiency of knowledge-based planning (KBP) using this highly refined model in improving the consistency and quality of the VMAT plans were also evaluated.

Results: The prediction ability was reinforced with the increased number of refinements in terms of normal tissue sparing. With enhanced prediction accuracy, more than $60 \%$ of automatic plan-6 (AP-6) plans (22/35) can be directly approved for clinical treatment without any manual revision. The plan quality scores for clinically approved plans (CPs) and manual plans (MPs) were on average 89.02 \pm 4.83 and $86.48 \pm 3.92(p<0.001)$. Knowledge-based planning significantly reduced the $D_{\text {mean }}$ and $V_{18 \text { Gy }}$ for kidney $(L / R)$, the $D_{\text {mean }} V_{30 ~ G y}$ and $V_{40 ~ G y}$ for bladder, rectum, and femoral head $(L / R)$.

Conclusion: The proposed model evolution method provides a practical way for the KBP to enhance its prediction ability with minimal human intervene. This highly refined prediction model can better guide KBP in improving the consistency and quality of the VMAT plans.

Keywords: Prediction model, Volumetric modulated arc therapy, Knowledge-based planning, Progressive training, Cervical cancer

*Correspondence: qizhy@sysucc.org.cn

${ }^{\dagger}$ Mingli Wang, Huikuan Gu and Jiang Hu contributed equally to this study

${ }^{1}$ Department of Radiation Oncology, Sun Yat-Sen University Cancer Center, Guangzhou, People's Republic of China

Full list of author information is available at the end of the article

\section{Introduction}

Volumetric modulated arc therapy (VMAT) followed by intracavitary brachytherapy has become one of major treatment modalities for cervical cancer [1-4]. However, developing an appropriate VMAT plan presents a real challenge, since inverse VMAT planning in essence is still a trial-and-error procedure. The planner has to manually set the starting optimization objectives for the tumor original author(s) and the source, provide a link to the Creative Commons licence, and indicate if changes were made. The images or other third party material in this article are included in the article's Creative Commons licence, unless indicated otherwise in a credit line to the material. If material is not included in the article's Creative Commons licence and your intended use is not permitted by statutory regulation or exceeds the permitted use, you will need to obtain permission directly from the copyright holder. To view a copy of this licence, visit http://creativecommons.org/licenses/by/4.0/. The Creative Commons Public Domain Dedication waiver (http://creativeco mmons.org/publicdomain/zero/1.0/) applies to the data made available in this article, unless otherwise stated in a credit line to the data. 
target as well as for each organ of interest, which needs to take into account the patient anatomy, the linac performance, the prescription doses and the organ dose tolerance limits. This makes VMAT planning operator- and experience-dependent, as too "easy" objectives may lead to suboptimal plan while too hard objectives may cause sub-optimal trade-offs. Several authors have reported some head \& neck and prostate cases suffering from over irradiation to the organs at risk (OAR) due to suboptimal treatment plans [5-7]. To address this issue, knowledgebased planning (KBP) arouses growing interest, which utilizes the prior knowledge to predict what kind of dose distribution is achievable and hence automatically generates the patient-specific optimization objectives for each OAR according to the estimated dose volume histograms (DVHs). Various KBP methods have been developed [8-14] and among them, RapidPlan ${ }^{\mathrm{TM}}$ is the first commercial software that has been put into clinical use. Previously published works have demonstrated its usefulness in improving plan quality and planning efficiency for tumors in head \& neck, prostate and rectum [15-19].

One of major concerns about the use of KBP is the quality of the plan database, which may determine the degree of accuracy that a prediction model can offer. It has already been revealed that current estimated results can only fulfil the "clinical acceptable" criteria rather than "optimal" or "near optimal" standards, due to the fact that the database plans may not all possess optimal dose distributions [5, 9]. Some researches tried to re-optimize each of prior plans by a group of experts to guarantee a high quality [20-22]. This is tremendously labor intensive and time consuming, especially for cases where a large number of training samples are used. To improve the predictive accuracy more efficiently, Appenzoller et al. introduced a refined method to take the estimated DVHs as a reference to exclude suboptimal plans from the training cohort and repeat the modeling process on the remaining training dataset [11]. Wang et al. demonstrated that both the prediction model and its constituent plans were able to be significantly improved after two runs of closed loop refinements [22]. More recently, a refined model has been applied in an ongoing multi-institutional clinical trial as a quality assurance tool, highlighting its great potential for accurate DVH prediction [21]. Nevertheless, as it has been supposed that the quality of the database can be improved over time by using the KBP method [ 5 , $9,13]$, this may suggest that the prediction model should also be retrained on a regular basis to ensure its predictive accuracy. Therefore, it was expected to develop a progressive training strategy striving to create a highly refined KBP model with minimum human intervene.

Another issue often encountered in model generation is how many patient plans are recommended to build a particular prediction model. The manufacturer recommended that the minimum number of plans required for RapidPlan model creation was 20 , but they emphasized that adding additional plans would usually help create a more robust model [23]. A newly published research concluded that the minimum required sample size needed to accurately train KBP models for prostate cancer depends on the specific model and endpoint to be predicted, and a sample size greater than 75 was recommended to train the KBP models [24]. Hence it is of primary importance to determine a propriate number of training samples in establishing the prediction model for cervical cancer to maintain its accuracy and robustness.

In this study, we present our experience in the application of KBP in VMAT treatment of cervical cancer with special attention to the above issues. A highly refined DVH prediction model was built for VMAT treatment of cervical cancer, which underwent a total of six runs of refining through progressive training until the training set size increased up to 100 cases. The proposed model evolution method was assessed in 35 new cervical cancer cases. The reliability and efficiency of KBP using this highly refined model in improving the consistency and quality of the VMAT plans were analyzed.

\section{Methods and materials Database}

A total of 100 patients with stage IA-IVB cervical cancer treated by pelvic VMAT were retrospectively reviewed. All patients were immobilized in the supine position. The $\mathrm{CT}$ images were acquired by a $\mathrm{CT}$ simulator using $3 \mathrm{~mm}$ slice and $3 \mathrm{~mm}$ spacing. The gross target volume (GTV) included all grossly enlarged lymph nodes with a short diameter of $\geq 1 \mathrm{~cm}$ and regional metastatic lymph nodes on imaging findings or as determined by PET/CT findings. The clinical target volume (CTV) included the cervix, whole uterus, parametrium, upper part of the vagina, and pelvic lymphatic drainage area (common, internal, and external iliac; obturator; and presacral). Inguinal lymph nodes were included if lower one-third vaginal involvement was observed. In patients with common iliac metastatic lymph nodes, para-aortic irradiation was administered. The planning gross target volume (PGTVnd) was generated by adding a $5-\mathrm{mm}$ margin to the GTV and the planning clinical target volume (PCTV) was generated by adding a 6-mm margin to the CTV in all orientations, except for the anterior direction where a $10-\mathrm{mm}$ margin was used. Dual-arc VMAT plans were designed by using Varian Eclipse treatment planning system (Varian Medical Systems, Palo Alto, CA), including two coplanar full arcs with gantry rotating counterclockwise from $179^{\circ}$ to $181^{\circ}$ and clockwise from $181^{\circ}$ to $179^{\circ}$. Dose prescription was set to be 60 Gy in 25 fractions to 
the PGTVnd and 45 Gy in 25 fractions to the PCTV. The planning goals for tumor targets and dose constraints for the OARs were detailed in Table 1. Recent follow-up indicated that all patients were proved to have favorable prognoses with neither severe late toxicity nor treatment failure (local recurrence/distant metastasis).

\section{Model building and evolution}

The prediction model was automatically generated for pelvic organs of interest, specific for each OAR, based on the principle of parameterization of the structure set and dose matrices for the prior plans in the training set. The built-in proprietary algorithm for the RapidPlan ${ }^{\text {TM }}$ (version 13.5, Varian Medical Systems, Palo Alto, CA) is largely inspired by the methodology described by Yuan et al. [25].

In this study, an in-house model evolution strategy was developed to enhance the prediction ability of the model by progressively upgrading the database with new higherquality plans and re-training the model. The developed model was initially built using 25 clinically approved VMAT plans for cervical cancer (model $\mathrm{C}_{0}$ ), which was the minimum number of treatment plans suggested by the product specialist. A closed-loop refinement process was conducted subsequently, in which relatively suboptimal plans were identified by comparing estimated DVHs

Table 1 Plan quality evaluation criteria. The planning goals for tumor target and dose constraints for OARs were listed

\begin{tabular}{|c|c|c|c|}
\hline & \multicolumn{2}{|l|}{ Criteria } & \multirow[t]{2}{*}{ Quality scores } \\
\hline & Acceptable & Excellent & \\
\hline \multirow[t]{5}{*}{ PGTVnd } & $V_{60 G y}(\%) \geq 97$ & $V_{60 G y}(\%)=100$ & $4.8 / 8$ \\
\hline & $V_{66 G y}(\%) \leq 10$ & $V_{66 G y}(\%) \leq 5$ & $4.8 / 8$ \\
\hline & $D_{\min }(G y) \geq 54.6$ & $D_{\min }(G y) \geq 55.8$ & $3 / 5$ \\
\hline & $\mathrm{Cl}(0-1):$ & As high as possible & $0-2$ \\
\hline & $\mathrm{HI}(0-1):$ & As low as possible & $0-2$ \\
\hline \multirow[t]{5}{*}{ PCTV } & $V_{45 G y}(\%) \geq 97$ & $V_{45 G y}(\%)=100$ & $4.8 / 8$ \\
\hline & $V_{49.5 \text { Gy }}(\%) \leq 20$ & $V_{49.5 \text { Gy }}(\%) \leq 10$ & $4.8 / 8$ \\
\hline & $D_{\min }(G y) \geq 40.95$ & $D_{\min }(G y) \geq 41.85$ & $3 / 5$ \\
\hline & $\mathrm{Cl}(0-1):$ & As high as possible & $0-2$ \\
\hline & HI (0-1): & As low as possible & $0-2$ \\
\hline Spinal cord & $D_{0.03 c c}(G y) \leq 45$ & $D_{0.03 c c}(G y) \leq 40$ & $6 / 10$ \\
\hline Bladder & $D_{35.0 \%}(G y) \leq 50$ & $\mathrm{D}_{35.0 \%}(\mathrm{~Gy}) \leq 45$ & $6 / 10$ \\
\hline Rectum & $\mathrm{D}_{60.0 \%}(\mathrm{~Gy}) \leq 45$ & $\mathrm{D}_{60.0 \%}(\mathrm{~Gy}) \leq 40$ & $6 / 10$ \\
\hline Kidney (L) & $V_{18 G y}(\%) \leq 32$ & $V_{18 G \mathrm{y}}(\%) \leq 20$ & $3 / 5$ \\
\hline Kidney (R) & $V_{18 G y}(\%) \leq 32$ & $V_{18 G y}(\%) \leq 20$ & $3 / 5$ \\
\hline \multirow[t]{2}{*}{ Femoral head (L) } & $V_{35 G y}(\%) \leq 50$ & $V_{35 G y}(\%) \leq 15$ & $1.8 / 3$ \\
\hline & $D_{0.03 c c}(G y) \leq 65$ & $D_{0.03 c c}(G y) \leq 50$ & $1.2 / 2$ \\
\hline \multirow[t]{2}{*}{ Femoral head (R) } & $V_{35 G y}(\%) \leq 50$ & $V_{35 G y}(\%) \leq 15$ & $1.8 / 3$ \\
\hline & $D_{0.03 c c}(G y) \leq 65$ & $D_{0.03 c c}(G y) \leq 50$ & $1.2 / 2$ \\
\hline Total score & / & / & 100 \\
\hline
\end{tabular}

with planned DVHs. Unlike previous studies [5, 9], these suboptimal plans were not excluded from the database, but were rejoined to the training dataset after they were re-optimized under the guidance of estimated DVHs to further spare the OARs. This resulted in a refined model $C_{1}$, preliminarily applied in clinic: (1) To guide the planning/re-planning process with better OAR sparing achieved; (2) To be a self-checking tool to identify the quality of the plan. By this means, VMAT plans with quality superior to the prediction were screened out and were added to the database to re-train the model on a monthly basis. Within the past 6 months, the developed model underwent five runs of refinement, generating model $C_{2}-C_{6}$, with training set size increased up to 100 cases. The detailed diagram of our model evolution process was illustrated in Fig. 1.

\section{Dosimetric evaluation}

The proposed model evolution method was assessed in 35 new cervical cancer cases. For comparison purpose, three kinds of VMAT plans were developed for each patient. (1) Automatic plan (AP): automatically created by only one click at the "optimization" button with no other human intervention; (2) Manual plan (MP): designed independently by a qualified planner in the traditional trial-and-error way; (3) Clinically approved plan $(\mathrm{CP})$ : created based on AP, but unlike AP, possible manual adjustments are permitted thereafter. The CPs were regarded as the reference standard in our plan comparison.

The prediction ability of the refined models of different stages was evaluated by comparing the estimated DVHs with the actual dose distributions finally achieved (i.e., DVHs derived from the CP, which was herein taken as the reference). This was done by assessing the degree of approximation between the predicted values and the reference values at given dosimetric endpoints. In consequence, the difference (in the absolute value) between the estimated dose and the reference dose of every model for a given OAR was calculated, and was ranked from small to large, with 6 points for the first, 5 points for the second, 4 points for the third, and so on. A total of 35 cases were evaluated and the average scores of various models were obtained for each OAR. The full mark of this investigation was 6 points. The introduction of such a scoring method is mainly to minimize the impact of individual cases on the global results.

To evaluate the usefulness of such a highly refined model in VMAT planning, a dosimetric comparison was conducted between APs generated by using model $C_{1}$ and model $\mathrm{C}_{6}$, respectively. The $\mathrm{CPs}$ generated based on model $\mathrm{C}_{6}$ were also compared with the MPs with respect to the target coverage, the OAR sparing and the planning 


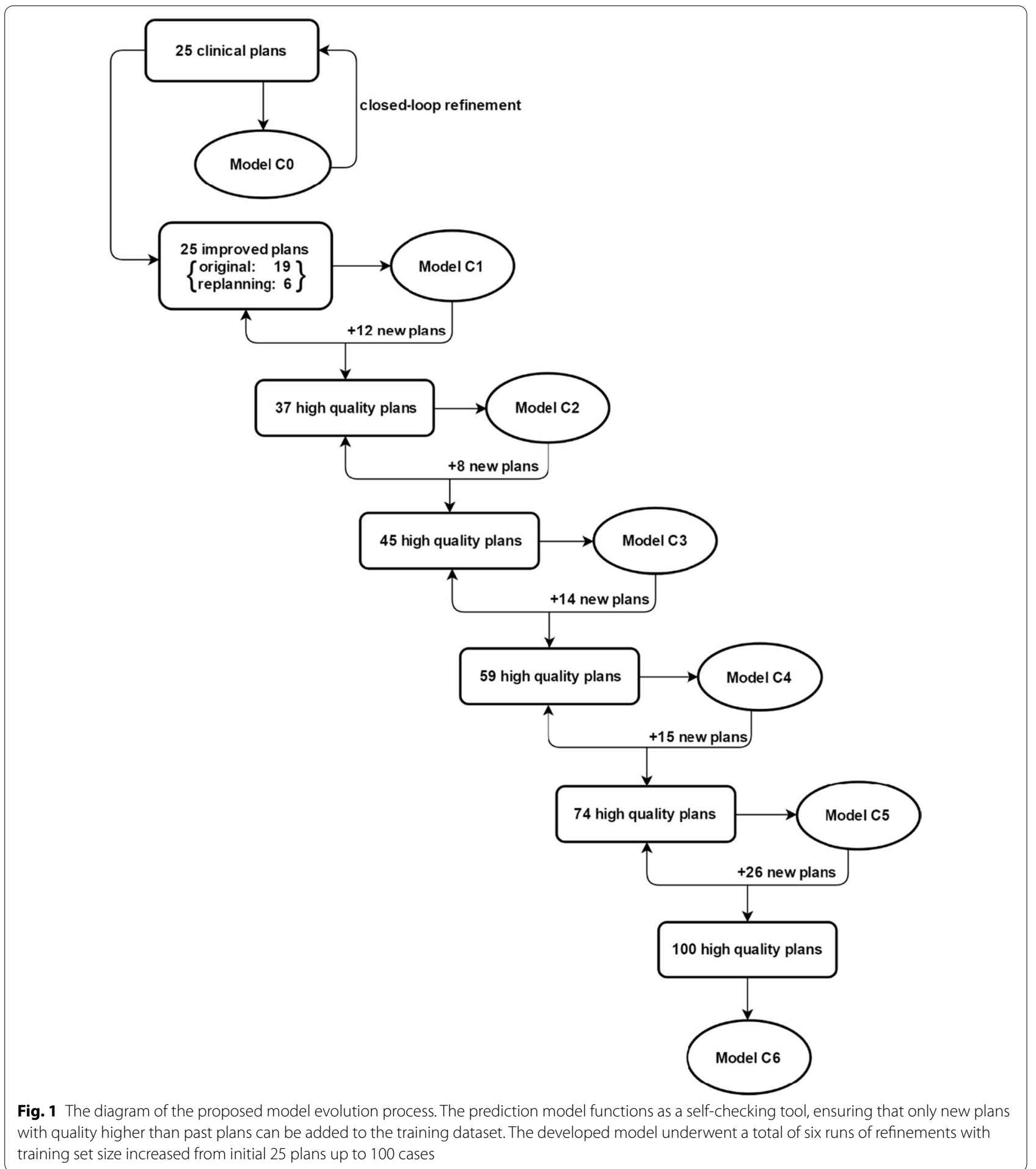

time. The effectiveness of KBP with this highly refined model in improving the consistency and quality of the VMAT plans were analyzed.
The dosimetric indices herein used for target dose evaluation include the dose coverage, the $\mathrm{CI}$ and HI. The conformity index (CI) [26] was calculated by: 


$$
\text { Conformity Index }(C I)=\left(\frac{V_{\text {Tref }}}{V_{T}}\right) \times\left(\frac{V_{\text {Tref }}}{V_{\text {ref }}}\right)
$$

where $\mathrm{V}_{\text {Tref }}$ refers to the volume of the target covered by the reference isodose (here $95 \%$ isodose), $\mathrm{V}_{T}$ was the target volume, and $\mathrm{V}_{\text {ref }}$ was the volume of the reference isodose (i.e., $98 \%$ isodose).

The homogeneity index (HI) was defined as follows:

$$
\text { Homogeneity Index }(H I)=\frac{D_{2 \%}-D_{98 \%}}{D_{50 \%}}
$$

where $D_{x \%}$ refers to the absorbed dose received by $x \%$ of the target volume [27].

The dosimetric indices to OARs were selected according to their radiobiological properties. The average dose $\left(D_{\text {mean }}\right)$ was computed for parallel organs, while the maximum dose $\left(D_{\max }\right)$ was recorded for serial organs like spinal cord. Other dosimetric indices collected include: $V_{18}$ Gy for kidney (L/R) and $V_{30 \text { Gy }}, V_{40 \text { Gy }}$ and $V_{50 \text { Gy }}$ for bladder, rectum, and femoral head (L/R).

To quantify the difference between plans, an assessing tool, namely Plan Quality Metric (PQM), was introduced $[28,29]$. The penalty points were assigned to the tumor target and each OAR, according to the priority of dose optimization objectives. The built-in dosimetric endpoints were determined with reference to our institutional protocols and the RTOG 0921 guideline [30]. The scoring details were listed in Table 1.

\section{Statistical analysis}

All statistical analyses were performed with SPSS software (version 20, SPSS Inc, Chicago, IL). The analyses of variance (ANOVA) were applied when normality (and homogeneity of variance) assumptions are satisfied. Otherwise, Wilcoxon Signed Rank test will be used. The statistically significant level was set as 0.05 .

\section{Results}

Figure 2 plotted the predicted doses, the actual doses, and the scores for different models of a certain organ of 35 cervical cancer patients. There is a tendency that the predictive accuracy was reinforced with the increased number of refinements, in terms of the degree of approximation of the predicted doses to the actual values. This can be more clearly seen in the scoring curve, which minimize the impact of individual cases on the global results by introducing the weighted scores. The predictive outcomes of model $C_{1}$ were relatively poor, most of which were ranked at the bottom and got the lowest score. Model $\mathrm{C}_{5}$ obtained a score approximate to 5 points in most cases, while model $\mathrm{C}_{6}$ provided the best estimate to the actual doses among the refined models of different stages. The associated scores for model $\mathrm{C}_{6}$ were all above
5.5 points for various tested OARs (The full mark is 6 points). It seems that the prediction ability approaches the limits of current planning skills (i.e., best effort plan) after five to six runs of re-training, when the training samples increases up to about 75 to 100 cases.

The refined model $\mathrm{C}_{1}$ and $\mathrm{C}_{6}$ were applied in automatic KBP for cervical cancer. Compared with APs created by model $C_{1}$ (AP-1), APs created by model $C_{6}$ (AP-6) shows advantages in dealing with the trade-offs between the target coverage and the dose to the OAR (Table 2). The proportion of AP- 6 that can directly satisfy the clinical requirements without any manual revision was $22 / 35$, while that of AP-1 was $16 / 35$. The plan quality scoring gave the average values of $85.61 \pm 6.78$ and $83.92 \pm 6.86$ for AP-6 and AP-1 $(\mathrm{p}=0.013)$.

The dosimetric results of CPs vs. MPs were given in Table 3. It was shown that both sets of VMAT plans achieved the dose coverage of $\mathrm{V}_{60 \mathrm{~Gy}}$ higher than $99 \%$ for PGTVnd and PCTV. Compared with MPs, CPs exhibited lower $\mathrm{V}_{110 \%}(p<0.001)$ and better CI $(p=0.001)$ for PCTV at a slight sacrifice of target dose coverage $(p=0.011)$ and minimal dose $\mathrm{D}_{\text {min }}(p=0.002)$. However, The $\mathrm{D}_{\text {min }}$ was all greater than $93 \%$ of the prescription dose for both kinds of treatment plans. The average plan quality scores for tumor target (PGTVnd plus PCTV) were $43.39 \pm 4.04$ and 42.23 \pm 3.47 for CP and MP $(p=0.011)$ (Table 4).

As for the radiation dose to OARs, CPs significantly reduced almost all the dosimetric indices except for the bladder $\mathrm{V}_{50 \mathrm{~Gy}}(\%)$ and the rectum $\mathrm{V}_{50 \mathrm{~Gy}}(\%)$, compared with MPs (Table 3). The overall quality assessment gave the mean scores of $89.02 \pm 4.83$ and $86.48 \pm 3.92$, respectively, for CPs and MPs $(p<0.001)$ (Table 4$)$.

\section{Discussion}

Although pelvic VMAT has been an increasingly used technique for treatment of cervical cancer, designing an appropriate VMAT plan remains a challenge. The major difficulty lies in the fact that the planner usually does not know what kind of dose distribution is achievable for each OAR. Due to limited planning time, especially in some busy centers, the planner may not have enough chance to repeatedly adjust the dose distributions to explore whether there are better results. This tends to lead to some suboptimal plans. It was shown by us and others $[31,32]$ that there were quite a few clinical plans that have room for improvement. Therefore, it has become a top priority to develop a way to improve the consistency and quality of the VMAT plans.

The KBP model can provide the estimated DVHs based on the prior knowledge, helping direct the planner's efforts towards an achievable high-quality plan. It was observed that the prediction ability was enhanced with the increased number of refinements in terms of 


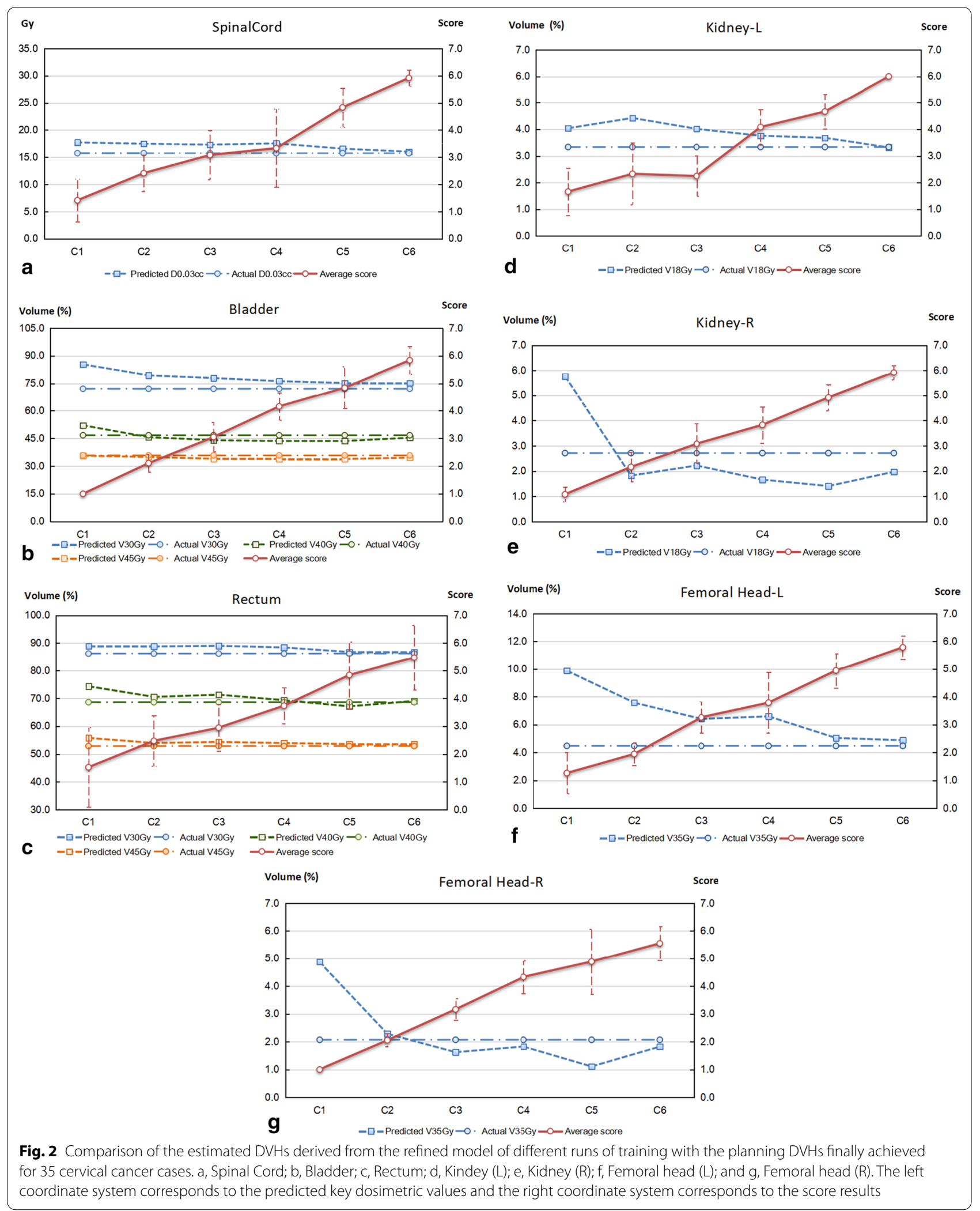


Table 2 Dosimetric comparison of AP-1 vs. AP-6. The statistical results between AP-1 scores and AP-6 scores were also given

\begin{tabular}{|c|c|c|c|c|c|c|}
\hline & & \multicolumn{2}{|l|}{$A P-1$} & \multicolumn{2}{|l|}{ AP-6 } & \multirow[t]{2}{*}{$p$ value } \\
\hline & & Results & Scores & Results & Scores & \\
\hline \multirow[t]{5}{*}{ PGTVnd } & $V_{60 G y}(\%)$ & $94.19 \pm 8.76$ & $4.11 \pm 2.77$ & $96.86 \pm 4.91$ & $5.46 \pm 2.73$ & $<0.001$ \\
\hline & $\mathrm{V}_{66 \mathrm{~Gy}}(\%)$ & $0.00 \pm 0.00$ & $8.00 \pm 0.00$ & $0.00 \pm 0.00$ & $8.00 \pm 0.00$ & / \\
\hline & $D_{\min }(G y)$ & $58.16 \pm 2.59$ & $4.76 \pm 0.92$ & $58.52 \pm 1.60$ & $4.80 \pm 0.77$ & 0.159 \\
\hline & $\mathrm{Cl}$ & $0.62 \pm 0.11$ & $1.24 \pm 0.21$ & $0.58 \pm 0.11$ & $1.16 \pm 0.22$ & $<0.001$ \\
\hline & $\mathrm{HI}$ & $0.05 \pm 0.02$ & $1.90 \pm 0.04$ & $0.05 \pm 0.02$ & $1.89 \pm 0.03$ & 0.240 \\
\hline \multirow[t]{5}{*}{ PCTV } & $V_{45 G y}(\%)$ & $95.96 \pm 6.94$ & $4.79 \pm 1.79$ & $98.55 \pm 3.16$ & $6.81 \pm 1.33$ & $<0.001$ \\
\hline & $\mathrm{V}_{49.5 \mathrm{~Gy}}(\%)$ & $13.67 \pm 6.42$ & $6.71 \pm 1.92$ & $18.90 \pm 6.83$ & $5.11 \pm 2.05$ & $<0.001$ \\
\hline & $D_{\min }(G y)$ & $36.72 \pm 10.69$ & $2.23 \pm 2.03$ & $38.01 \pm 10.79$ & $3.05 \pm 2.17$ & 0.008 \\
\hline & $\mathrm{Cl}$ & $0.84 \pm 0.03$ & $1.68 \pm 0.06$ & $0.82 \pm 0.02$ & $1.65 \pm 0.04$ & $<0.001$ \\
\hline & $\mathrm{HI}$ & $0.32 \pm 0.06$ & $1.37 \pm 0.12$ & $0.30 \pm 0.06$ & $1.40 \pm 0.11$ & 0.095 \\
\hline Spinal cord & $\mathrm{D}_{0.03 c c}(\mathrm{~Gy})$ & $14.13 \pm 12.49$ & $10.00 \pm 0.00$ & $14.38 \pm 13.44$ & $10.00 \pm 0.00$ & / \\
\hline Bladder & $\mathrm{D}_{35 \%}(\mathrm{~Gy})$ & $42.84 \pm 8.13$ & $9.50 \pm 0.61$ & $43.54 \pm 8.10$ & $9.18 \pm 0.88$ & $<0.001$ \\
\hline Rectum & $\mathrm{D}_{60.0 \%}(\mathrm{~Gy})$ & $41.54 \pm 7.47$ & $7.72 \pm 1.44$ & $42.43 \pm 7.63$ & $7.40 \pm 1.65$ & $<0.001$ \\
\hline Kidney (L) & $\mathrm{V}_{18 \mathrm{~Gy}}(\%)$ & $3.05 \pm 4.71$ & $5.00 \pm 0.00$ & $2.90 \pm 4.60$ & $5.00 \pm 0.00$ & / \\
\hline Kidney (R) & $\mathrm{V}_{18 \mathrm{~Gy}}(\%)$ & $3.16 \pm 7.19$ & $4.91 \pm 0.53$ & $2.32 \pm 3.88$ & $5.00 \pm 0.00$ & 0.324 \\
\hline \multirow[t]{2}{*}{ Femoral head (L) } & $V_{35 G y}(\%)$ & $7.80 \pm 3.80$ & $3.00 \pm 0.02$ & $5.59 \pm 3.29$ & $3.00 \pm 0.00$ & 0.211 \\
\hline & $\mathrm{D}_{0.03 c c}(\mathrm{~Gy})$ & $40.95 \pm 7.47$ & $2.00 \pm 0.00$ & $41.12 \pm 7.61$ & $2.00 \pm 0.00$ & / \\
\hline \multirow[t]{2}{*}{ Femoral head (R) } & $\mathrm{V}_{35 \mathrm{~Gy}}(\%)$ & $5.55 \pm 4.79$ & $2.96 \pm 0.17$ & $3.70 \pm 4.96$ & $2.96 \pm 0.18$ & 0.879 \\
\hline & $\mathrm{D}_{0.03 c c}(\mathrm{~Gy})$ & $38.98 \pm 7.74$ & $2.00 \pm 0.00$ & $39.48 \pm 7.63$ & $2.00 \pm 0.00$ & / \\
\hline Total score & & & $83.92 \pm 6.86$ & & $85.61 \pm 6.78$ & 0.013 \\
\hline
\end{tabular}

AP refers to the fully automatic plan, which was created by only one click at the "optimization" button with no other human intervention. AP- 1 and AP- 6 were fully automatic plans generated by using model $C_{1}$ and model $C_{6}$, respectively

OAR sparing for most OARs. This may result from the joint actions of the increased number of training samples and the improved quality of the plan database. Currently, there are few studies related to the required training samples size for a particular KBP model. The manufacturer specialists suggested that the minimum number of plans required for RapidPlan model creation was 20 , but they also emphasized that adding additional plans would usually help create a more robust model [23]. Meanwhile, a newly published research discussed that although only 20 samples were needed to predict the rectum DVH, a sample size greater than 75 was recommended to train the KBP model [24]. This is why we started training from 25 samples and finally increased the sample size up to 100 cases. Our experiments proved once again the advantages of large training sample size in establishing the prediction model. By continuously updating the database with new plans of higher quality than before, the quality of the database was improved over time in a systematic way, which had interactive impact on the KBP model. The prediction model experiencing several runs of progressive training was found to provide better estimates for the final dose distribution.

With enhanced prediction ability, the highly refined model has shown its advantage in capturing actual clinical practices during the knowledge-based VMAT planning of cervical cancer. More than $60 \%$ of AP- 6 plans can be directly approved for clinical treatment. The primary reason for the failure of automatic planning is the insufficient coverage of PCTV by the prescription dose in the overlapping region of PCTV to rectum and PCTV to bladder. There is a tendency for pelvic radiotherapy in our practice that the high dose coverage of tumor target area will be improved preferentially to ensure local tumor control when the radiation doses of critical organs do not exceed the dose tolerance limits. However, the RapidPlan takes the lower bound of the DVH estimate range as the optimization objectives by default with attempt to maximize OAR sparing. This, on the other hand, may lead to underdose of the adjacent tumor target. Adding a 3-mm ring structure outside the PCTV to allow for the high dose fall-off helps improve the success rate of automatic planning. More research is warranted.

Compared with the conventional trial-and-error planning method, our results demonstrated that the novel KBP method could enhance the quality of treatment planning in term of better OAR protection. Radiation induced acute and chronic toxicities, including small bowel obstruction, enteritis, proctitis, and radiation cystitis are serious issue of concern directly related to 
Table 3 Dosimetric results for two kinds of VMAT plans. The corresponding statistical analyses were also included

\begin{tabular}{|c|c|c|c|c|}
\hline & & $\mathrm{CP}$ & MP & $p$ value \\
\hline \multirow[t]{5}{*}{ PGTVnd } & $\mathrm{V}_{60 \mathrm{~Gy}}(\%)$ & $99.36 \pm 0.59$ & $99.40 \pm 0.71$ & 0.785 \\
\hline & $\mathrm{V}_{66 \mathrm{~Gy}}(\%)$ & $0.15 \pm 0.53$ & $0.08 \pm 0.35$ & 0.095 \\
\hline & $D_{\min }(G y)$ & $59.12 \pm 1.01$ & $59.35 \pm 0.73$ & 0.131 \\
\hline & $\mathrm{Cl}$ & $0.54 \pm 0.11$ & $0.54 \pm 0.10$ & 0.717 \\
\hline & $\mathrm{HI}$ & $0.05 \pm 0.01$ & $0.05 \pm 0.01$ & 0.444 \\
\hline \multirow[t]{5}{*}{ PCTV } & $V_{45 G y}(\%)$ & $99.48 \pm 0.58$ & $99.73 \pm 0.20$ & 0.011 \\
\hline & $\mathrm{V}_{49.5 \mathrm{~Gy}}(\%)$ & $16.04 \pm 9.50$ & $24.43 \pm 12.16$ & $<0.001$ \\
\hline & $D_{\min }(G y)$ & $38.44 \pm 10.87$ & $39.49 \pm 11.10$ & 0.002 \\
\hline & $\mathrm{Cl}$ & $0.81 \pm 0.03$ & $0.79 \pm 0.03$ & 0.001 \\
\hline & $\mathrm{HI}$ & $0.30 \pm 0.06$ & $0.30 \pm 0.06$ & 0.875 \\
\hline Spinal cord & $\mathrm{D}_{0.03 c c}(\mathrm{~Gy})$ & $14.72 \pm 13.67$ & $15.42 \pm 14.28$ & 0.019 \\
\hline \multirow[t]{5}{*}{ Bladder } & $D_{\text {mean }}(c G y)$ & $3791.45 \pm 704.91$ & $4076.11 \pm 743.14$ & $<0.001$ \\
\hline & $\mathrm{D}_{35 \%}(\mathrm{~Gy})$ & $43.79 \pm 8.18$ & $44.66 \pm 7.95$ & 0.004 \\
\hline & $\mathrm{V}_{30}(\%)$ & $75.92 \pm 16.59$ & $89.66 \pm 18.34$ & $<0.001$ \\
\hline & $V_{40}(\%)$ & $50.20 \pm 15.19$ & $66.03 \pm 17.42$ & $<0.001$ \\
\hline & $V_{50}(\%)$ & $3.67 \pm 3.40$ & $2.27 \pm 2.72$ & 0.003 \\
\hline \multirow[t]{5}{*}{ Rectum } & $\mathrm{D}_{\text {mean }}(\mathrm{cGy})$ & $4189.89 \pm 751.59$ & $4301.27 \pm 759.75$ & $<0.001$ \\
\hline & $\mathrm{D}_{60 \%}(\mathrm{~Gy})$ & $43.09 \pm 7.82$ & $44.31 \pm 7.82$ & 0.001 \\
\hline & $V_{30}(\%)$ & $90.46 \pm 16.36$ & $93.09 \pm 16.73$ & $<0.001$ \\
\hline & $V_{40}(\%)$ & $75.40 \pm 16.40$ & $84.76 \pm 16.64$ & $<0.001$ \\
\hline & $\mathrm{V}_{50}(\%)$ & $1.87 \pm 3.79$ & $1.24 \pm 3.55$ & 0.277 \\
\hline \multirow[t]{2}{*}{ Kidney-L } & $\mathrm{D}_{\text {mean }}(\mathrm{cGy})$ & $427.02 \pm 416.72$ & $488.35 \pm 486.71$ & 0.001 \\
\hline & $V_{18}(\%)$ & $3.22 \pm 5.05$ & $4.73 \pm 7.19$ & 0.019 \\
\hline \multirow[t]{2}{*}{ Kidney-R } & $D_{\text {mean }}(c G y)$ & $472.90 \pm 390.95$ & $575.53 \pm 483.65$ & $<0.001$ \\
\hline & $V_{18}(\%)$ & $2.65 \pm 4.53$ & $6.42 \pm 7.10$ & $<0.001$ \\
\hline \multirow[t]{6}{*}{ Femoral-L } & $D_{\text {mean }}(c G y)$ & $1667.98 \pm 438.95$ & $2164.23 \pm 541.71$ & $<0.001$ \\
\hline & $\mathrm{D}_{0.03 c c}(\mathrm{~Gy})$ & $41.23 \pm 7.68$ & $43.94 \pm 7.76$ & $<0.001$ \\
\hline & $V_{30}(\%)$ & $12.82 \pm 6.76$ & $26.61 \pm 14.25$ & $<0.001$ \\
\hline & $\mathrm{V}_{35}(\%)$ & $5.91 \pm 3.58$ & $15.94 \pm 8.77$ & $<0.001$ \\
\hline & $V_{40}(\%)$ & $1.25 \pm 1.54$ & $6.24 \pm 3.81$ & $<0.001$ \\
\hline & $V_{50}(\%)$ & / & / & / \\
\hline \multirow[t]{6}{*}{ Femoral-R } & $\mathrm{D}_{\text {mean }}(\mathrm{cGy})$ & $1551.25 \pm 432.40$ & $2117.46 \pm 571.05$ & $<0.001$ \\
\hline & $\mathrm{D}_{0.03 c \mathrm{cc}}(\mathrm{Gy})$ & $39.70 \pm 7.73$ & $43.43 \pm 7.84$ & $<0.001$ \\
\hline & $V_{30}(\%)$ & $9.32 \pm 8.71$ & $24.51 \pm 16.05$ & $<0.001$ \\
\hline & $V_{35}(\%)$ & $4.01 \pm 5.41$ & $14.91 \pm 13.59$ & $<0.001$ \\
\hline & $\mathrm{V}_{40}(\%)$ & $0.89 \pm 2.04$ & $6.25 \pm 8.76$ & $<0.001$ \\
\hline & $\mathrm{V}_{50}(\%)$ & / & / & / \\
\hline
\end{tabular}

MP refers to the manual plan. CP refers to the clinically approved plan, which was created based on knowledge-based planning with possible manual adjustments thereafter. The CP was taken as our reference standard in this study

the quality of life. It has been reported that the incident rates of grade 3 or higher complications in 83 patients treated with pelvic IMRT plus high dose rate brachytherapy are $2.4 \%$ and $3.6 \%$ for the rectum and the bladder, respectively [1]. By applying KBP, the dosimetric indices of the rectum and the bladder, such as $D_{\text {mean }}, V_{40 G y}$ and $\mathrm{V}_{30 \text { Gy }}$, were all significantly reduced $(p<0.001)$ under the
Table 4 Plan quality scores for CPs and MPs. The corresponding statistical analyses were included

\begin{tabular}{|c|c|c|c|c|}
\hline & & $\mathrm{CP}$ & MP & $p$ value \\
\hline \multirow[t]{5}{*}{ PGTVnd } & $\mathrm{V}_{60 \mathrm{~Gy}}(\%)$ & $7.31 \pm 0.63$ & $7.36 \pm 0.76$ & 0.784 \\
\hline & $\mathrm{V}_{66 \mathrm{~Gy}}(\%)$ & $8.00 \pm 0.00$ & $8.00 \pm 0.00$ & / \\
\hline & $D_{\min }(G y)$ & $4.98 \pm 0.12$ & $5.00 \pm 0.00$ & 0.324 \\
\hline & $\mathrm{Cl}$ & $1.07 \pm 0.21$ & $1.08 \pm 0.20$ & 0.679 \\
\hline & $\mathrm{HI}$ & $1.90 \pm 0.03$ & $1.90 \pm 0.02$ & 0.473 \\
\hline \multirow[t]{5}{*}{ PCTV } & $V_{45 G y}(\%)$ & $7.44 \pm 0.61$ & $7.71 \pm 0.21$ & 0.011 \\
\hline & $\mathrm{V}_{49.5 \mathrm{~Gy}}(\%)$ & $6.04 \pm 2.71$ & $4.04 \pm 2.56$ & $<0.001$ \\
\hline & $D_{\min }(G y)$ & $3.62 \pm 1.97$ & $4.17 \pm 1.60$ & 0.025 \\
\hline & $\mathrm{Cl}$ & $1.62 \pm 0.05$ & $1.57 \pm 0.07$ & 0.001 \\
\hline & $\mathrm{HI}$ & $1.40 \pm 0.12$ & $1.40 \pm 0.13$ & 0.936 \\
\hline Spinal cord & $D_{0.03 c c}(G y)$ & $10.00 \pm 0.00$ & $10.00 \pm 0.00$ & / \\
\hline Bladder & $D_{35 \%}(G y)$ & $9.03 \pm 0.97$ & $8.90 \pm 0.85$ & 0.102 \\
\hline Rectum & $D_{60 \%}(G y)$ & $6.60 \pm 1.95$ & $5.62 \pm 1.40$ & $<0.001$ \\
\hline Kidney (L) & $\mathrm{V}_{18 \mathrm{~Gy}}(\%)$ & $5.00 \pm 0.00$ & $4.97 \pm 0.18$ & 0.315 \\
\hline Kidney (R) & $\mathrm{V}_{18 \mathrm{~Gy}}(\%)$ & $5.00 \pm 0.00$ & $4.98 \pm 0.09$ & 0.324 \\
\hline \multirow[t]{2}{*}{ Femoral head (L) } & $V_{35 G y}(\%)$ & $3.00 \pm 0.01$ & $2.89 \pm 0.24$ & 0.009 \\
\hline & $D_{0.03 c c}(G y)$ & $2.00 \pm 0.00$ & $2.00 \pm 0.00$ & / \\
\hline \multirow[t]{2}{*}{ Femoral head (R) } & $V_{35 G y}(\%)$ & $2.96 \pm 0.18$ & $2.85 \pm 0.45$ & 0.081 \\
\hline & $D_{0.03 c c}(G y)$ & $2.00 \pm 0.00$ & $2.00 \pm 0.00$ & / \\
\hline Total score & & $89.02 \pm 4.83$ & $86.48 \pm 3.92$ & $<0.001$ \\
\hline
\end{tabular}

condition of approximately similar target dose distributions. This may contribute to the fact that the incidence of late toxicities at our institution appeared to be lower than those reported in previous studies [3]. Moreover, the KBP method was found to help standardize the patient treatment, making treatment results less operator- and experience-dependent. In dosimetric comparisons, even CPs designed by a junior planner can achieve dose distributions comparable to MPs. Notably, the time spent for a KBP plan is much lower than that for a manual plan, even if manual revision is required.

\section{Conclusion}

The proposed model evolution method not only utilizes the KBP model to guide the planning process, but also takes it as a self-checking tool to identify high quality plans, providing a practical way to enhance the prediction ability with minimal human intervene. It has proved to our satisfaction that this highly refined prediction model can better guide KBP in improving the consistency and quality of the VMAT plans. The method described here was universal and can be used for some other cancer sites. However, in order to satisfy the diverse needs of clinical practice, it is recommended that each unit establishes its own model using this refinement method. 


\section{Acknowledgements}

Many people have offered us valuable help in our study. We would like to give our sincere gratitude to Dr. Ouyang and Prof. Cao, who gave us great help by providing clinical materials. Moreover, we pleased to acknowledge Mr. Xie for his invaluable assistant in statistical analysis.

\section{Authors' contributions}

Zhenyu Qi designed and supervised the study. Zhenyu Qi, Mingli Wang, Huikuan Gu and Jiang Hu developed the automatic KBP method, collected and analyzed the data. Jian Liang and Sisi Xu provided technical assistance for the study. Zhenyu Qi, Mingli Wang, Huikuan Gu and Jiang Hu wrote the manuscript. All co-authors have reviewed and approved this version of the manuscript. All authors read and approved the final manuscript.

\section{Funding}

This work was supported in part by National Natural Science Foundation of China, No. 81371710; Science and technology Program of Guangdong Province, China, No. 2013B021800149; and Science and Technology Program of Guangzhou, China, No. 201607010199.

\section{Availability of supporting data}

The datasets are backed up on the Research Data Deposit (RDD Number: RDDA2020001629, https://www.researchdata.org.cn) and are available upon reasonable request.

\section{Declarations}

\section{Ethical approval and consent to participate}

Our study was reviewed and approved by the IRB committee of Sun Yat-sen University Cancer Center, with the approval number of B2019-131-01. Written informed consent was obtained from the participants of this study.

\section{Consent for publication}

Not applicable.

\section{Competing interests}

The authors indicate no actual or potential competing interest exist.

\section{Author details}

${ }^{1}$ Department of Radiation Oncology, Sun Yat-Sen University Cancer Center, Guangzhou, People's Republic of China. ${ }^{2}$ State Key Laboratory of Oncology in South China, Collaborative Innovation Center for Cancer Medicine, Guangzhou, People's Republic of China. ${ }^{3}$ Guangdong Key Laboratory of Nasopharyngeal Carcinoma Diagnosis and Therapy, Guangzhou, People's Republic of China.

Received: 21 December 2020 Accepted: 10 March 2021

Published online: 22 March 2021

\section{References}

1. Chen SW, Liang JA, Hung YC, Yeh LS, Chang WC, Lin WC, et al. Does initial $45 \mathrm{~Gy}$ of pelvic intensity-modulated radiotherapy reduce late complications in patients with locally advanced cervical cancer? A cohort control study using definitive chemoradiotherapy with high-dose rate brachytherapy. Radiol Oncol. 2013;47:176-84. https://doi.org/10.2478/ raon-2013-0011.

2. Vulpe H, Asamoah FA, Maganti M, Vanderpuye V, Fyles A, Yarney J. External beam radiation therapy and brachytherapy for cervical cancer: the experience of the national centre for radiotherapy in Accra, Ghana. Int J Radiat Oncol Biol Phys. 2018;100:1246-53. https://doi.org/10.1016/j. ijrobp.2017.12.270.

3. Lin Y, Ouyang Y, Chen K, Lu Z, Liu Y, Cao X. Clinical outcomes of volumetric modulated arc therapy following intracavitary/interstitial brachytherapy in cervical cancer: a single institution retrospective experience. Front Oncol. 2019;9:760. https://doi.org/10.3389/fonc.2019.00760.

4. Quan EM, Li X, Li Y, Wang X, Kudchadker RJ, Johnson JL, et al. A comprehensive comparison of IMRT and VMAT plan quality for prostate cancer treatment. Int J Radiat Oncol Biol Phys. 2012;83:1 169-78. https://doi.org/ 10.1016/j.jijobp.2011.09.015.

5. Wu B, Ricchetti F, Sanguineti G, Kazhdan M, Simari P, Chuang M, et al. Patient geometry-driven information retrieval for IMRT treatment plan quality control. Med Phys. 2009;36:5497-505. https://doi.org/10.1118/1. 3253464.

6. Moore KL, Brame RS, Low DA, Mutic S. Experience-based quality control of clinical intensity-modulated radiotherapy planning. Int J Radiat Oncol Biol Phys. 2011;81:545-51. https://doi.org/10.1016/j.jijobp.2010.11.030.

7. Zhu X, Ge Y, Li T, Thongphiew D, Yin FF, Wu QJ. A planning quality evaluation tool for prostate adaptive IMRT based on machine learning. Med Phys. 2011;38:719-26. https://doi.org/10.1118/1.3539749.

8. Zhang X, Li X, Quan EM, Pan X, Li Y. A methodology for automatic intensity-modulated radiation treatment planning for lung cancer. Phys Med Biol. 2011;56:3873-93. https://doi.org/10.1088/0031-9155/56/13/ 009.

9. Wu B, Ricchetti F, Sanguineti G, Kazhdan M, Simari P, Jacques R, et al. Data-driven approach to generating achievable dose-volume histogram objectives in intensity-modulated radiotherapy planning. Int J Radiat Oncol Biol Phys. 2011;79:1241-7. https://doi.org/10.1016/j.jprobp.2010.05. 026.

10. Petit SF, Wu B, Kazhdan M, Dekker A, Simari P, Kumar R, et al. Increased organ sparing using shape-based treatment plan optimization for intensity modulated radiation therapy of pancreatic adenocarcinoma. Radiother Oncol. 2011;102:38-44. https://doi.org/10.1016/j.radonc.2011. 05.025 .

11. Appenzoller LM, Michalski JM, Thorstad WL, Mutic S, Moore KL. Predicting dose-volume histograms for organs-at-risk in IMRT planning. Med Phys. 2012;39:7446-61. https://doi.org/10.1118/1.4761864.

12. Wu B, Pang D, Simari P, Taylor R, Sanguineti G, McNutt T. Using overlap volume histogram and IMRT plan data to guide and automate VMAT planning: a head-and-neck case study. Med Phys. 2013;40:02714. https:// doi.org/10.1118/1.4788671.

13. Wang Y, Zolnay A, Incrocci L, Joosten H, McNutt T, Heijmen B, et al. A quality control model that uses PTV-rectal distances to predict the lowest achievable rectum dose, improves IMRT planning for patients with prostate cancer. Radiother Oncol. 2013;107:352-7. https://doi.org/10.1016/j. radonc.2013.05.032

14. Zarepisheh M, Long T, Li N, Tian Z, Romeijn HE, Jia X, et al. A DVH-guided IMRT optimization algorithm for automatic treatment planning and adaptive radiotherapy replanning. Med Phys. 2014;41:061711. https://doi. org/10.1118/1.4875700.

15. Tol JP, Delaney AR, Dahele M, Slotman BJ, Verbakel WF. Evaluation of a knowledge-based planning solution for head and neck cancer. Int J Radiat Oncol Biol Phys. 2015;91:612-20. https://doi.org/10.1016/j.ijrobp. 2014.11.014.

16. Chang ATY, Hung AWM, Cheung FWK, Lee MCH, Chan OSH, Philips H, et al. Comparison of planning quality and efficiency between conventional and knowledge-based algorithms in nasopharyngeal cancer patients using intensity modulated radiation therapy. Int J Radiat Oncol Biol Phys. 2016;95:981-90. https://doi.org/10.1016/j.jprobp.2016.02.017.

17. Kubo K, Monzen H, Ishii K, Tamura M, Kawamorita R, Sumida I, et al. Dosimetric comparison of RapidPlan and manually optimized plans in volumetric modulated arc therapy for prostate cancer. Physica Med. 2017:44:199-204. https://doi.org/10.1016/j.ejmp.2017.06.026.

18. Wu H, Jiang F, Yue H, Li S, Zhang Y. A dosimetric evaluation of knowledgebased VMAT planning with simultaneous integrated boosting for rectal cancer patients. J Appl Clin Med Phys. 2016;17:78-85. https://doi.org/10. 1120/jacmp.v17i6.6410.

19. Castriconi R, Fiorino C, Passoni P, Broggi S, Di Muzio NG, Cattaneo GM, et al. Knowledge-based automatic optimization of adaptive early-regression-guided VMAT for rectal cancer. Physica Med. 2020;70:58-64. https:// doi.org/10.1016/j.ejmp.2020.01.016.

20. Shiraishi S, Tan J, Olsen LA, Moore KL. Knowledge-based prediction of plan quality metrics in intracranial stereotactic radiosurgery. Med Phys. 2015;42:908-17. https://doi.org/10.1118/1.4906183.

21. Li N, Carmona R, Sirak I, Kasaova L, Followill D, Michalski J, et al. Highly efficient training, refinement, and validation of a knowledge-based planning quality-control system for radiation therapy clinical trials. Int J Radiat Oncol Biol Phys. 2017;97:164-72. https://doi.org/10.1016/j.jprobp.2016.10. 005. 
22. Wang M, Li S, Huang Y, Yue H, Li T, Wu H, et al. An interactive plan and model evolution method for knowledge-based pelvic VMAT planning. J Appl Clin Med Phys. 2018;19:491-8. https://doi.org/10.1002/acm2.12403.

23. Varian Website: https://www.myvarian.com/s/login/

24. Boutilier JJ, Craig T, Sharpe MB, Chan TC. Sample size requirements for knowledge-based treatment planning. Med Phys. 2016;43:1212-21. https://doi.org/10.1118/1.4941363.

25. Yuan L, Ge Y, Lee WR, Yin FF, Kirkpatrick JP, Wu QJ. Quantitative analysis of the factors which affect the interpatient organ-at-risk dose sparing variation in IMRT plans. Med Phys. 2012;39:6868-78. https://doi.org/10.1118/1. 4757927.

26. Feuvret L, Noël G, Mazeron JJ, Bey P. Conformity index: a review. Int J Radiat Oncol Biol Phys. 2006;64:333-42. https://doi.org/10.1016/j.jjrobp. 2005.09.028.

27. Yoon M, Park SY, Shin D, Lee SB, Pyo HR, Kim DY, et al. A new homogeneity index based on statistical analysis of the dose-volume histogram. J Appl Clin Med Phys. 2007;8:9. https://doi.org/10.1120/jacmp.v8i2.2390.

28. Nelms BE, Robinson G, Markham J, Velasco K, Boyd S, Narayan S, et al. Variation in external beam treatment plan quality: An inter-institutional study of planners and planning systems. Practical Radiation Oncology. 2012;2:296-305. https://doi.org/10.1016/j.prro.2011.11.012.

29. Fusella M, Scaggion A, Pivato N, Rossato MA, Zorz A, Paiusco M. Efficiently train and validate a RapidPlan model through APQM scoring. Med Phys. 2018:45:2611-9. https://doi.org/10.1002/mp.12896.
30. Viswanathan AN, Moughan J, Miller BE, Xiao Y, Jhingran A, Portelance L, et al. NRG Oncology/RTOG 0921: A phase 2 study of postoperative intensity-modulated radiotherapy with concurrent cisplatin and bevacizumab followed by carboplatin and paclitaxel for patients with endometrial cancer. Cancer. 2015;121:2156-63. https://doi.org/10.1002/cncr.29337.

31. Chung HT, Lee B, Park E, Lu JJ, Xia P. Can all centers plan intensity-modulated radiotherapy (IMRT) effectively? An external audit of dosimetric comparisons between tree-dimensional conformal radiotherapy and IMRT for adjuvant chemoradiation for gastric cancer. Int J Radiat Oncol Biol Phys. 2008;71:1167-74. https://doi.org/10.1016/j.jprobp.2007.11.040.

32. Williams MJ, Bailey MJ, Forstner D, Metcalfe PE. Multicentre quality assurance of intensity-modulated radiation therapy plans: a precursor to clinical trials. J Med Imag Radiat Oncol. 2010;51:472-9. https://doi.org/10. $1111 / j .1440-1673.2007 .01873 . x$

\section{Publisher's Note}

Springer Nature remains neutral with regard to jurisdictional claims in published maps and institutional affiliations.
Ready to submit your research? Choose BMC and benefit from:

- fast, convenient online submission

- thorough peer review by experienced researchers in your field

- rapid publication on acceptance

- support for research data, including large and complex data types

- gold Open Access which fosters wider collaboration and increased citations

- maximum visibility for your research: over $100 \mathrm{M}$ website views per year

At BMC, research is always in progress.

Learn more biomedcentral.com/submissions 